\title{
Is poverty a risk factor for CKD?
}

S hockwaves reverberated worldwide last September when it became apparent that Chinese milk supplies had been deliberately spiked with the toxic industrial chemical melamine to increase their apparent protein content. The heavy toll of the deception-six deaths and an estimated 300,000 cases of kidney stones and renal failure-was exacted largely on children from families in poor and remote areas of the country, for whom the low price of the adulterated milk products was especially attractive. The scandal serves as a tragic reminder of the vulnerability of people living in poverty to kidney damage.

In this month's Case Study, Suma Prakash and colleagues describe the detection and treatment of lead poisoning from an Ayurvedic herbal remedy in a South Asian man with chronic kidney disease (CKD). Traditional remedies are widely used in many developing countries, where expensive Western drugs are often unavailable and conventional medicine might be regarded with suspicion. Many such remedies-even those that are, like the lead-based Ayurvedic remedy, reputed to benefit the kidney-have acutely or chronically nephrotoxic effects. Infections that can be readily treated or prevented in the West, such as tuberculosis, are also major causes of kidney disease in developing countries. Rates of acute postinfectious glomerulonephritis, for example, are not declining in the developing world in the same way as they are in most industrialized nations, as Kanjanabuch and co-workers from Thailand discuss elsewhere in this issue.

Many developing countries are currently experiencing massive rates of economic growth; unfortunately, this growth is often accompanied by the adoption of unhealthy Western lifestyles at a pace that outstrips that of improvements in healthcare. Physical inactivity, consumption of cheap, energy-dense food, excessive alcohol intake and smoking impose a heavy burden on developing regions, where public awareness of the dangers they pose to health is limited. Tobacco use, for example, is growing exponentially in China, where the number of smokers currently exceeds the total US population. Western tobacco firms have been accused of contributing to this smoking epidemic by the use of aggressive promotional strategies.

Lifestyle factors make a huge contribution to the development of diabetes and hypertension, which are the main causes of kidney disease worldwide. The prevalence of hypertension is projected to increase by a sobering $80 \%$ in developing regions by 2025 ; the number of people with diabetes is expected to reach 228 million in developing countries by 2030. Clearly, the historical association of these conditions with affluence no longer holds true. Diabetes and hypertension are themselves a result of growing rates of overweight and obesity-which, paradoxically, are now counted alongside underweight and malnutrition as major threats to the health of the developing world.

The specter of kidney disease looms so large over the developing world that some regions are even identified with their own particular types of CKD. Over the past 5 years, a mysterious epidemic of CKD has affected more than 6,000 Sri Lankans, mostly male agricultural workers living in the Northern and Central regions of the country. Diabetes and hypertension have been ruled out as causative factors of the epidemic, leaving baffled physicians to consider the potential contribution of nephrotoxic pesticides or heavy metals. The etiology of Balkan endemic nephropathy, which exclusively affects communities along the Danube River, is similarly unclear.

According to the World Bank, approximately 2.7 billion people worldwide currently live in poverty, and this number will only increase as the global economic crisis deepens. Of course, poverty is not confined to poor countries: living on the breadline reduces access to healthcare in rich countries just as it does elsewhere, with the same end result on the kidney. As this year's World Kidney Day emphasized, the solution to the burgeoning epidemic of CKD among the poor is obvious: education, prevention and screening. The sheer scale of the problem demands that these measures are implemented in innovative and workable ways. In China, for example, where high salt intake is a major contributor to hypertension and cardiovascular disease, substitution with a reduced-sodium alternative has shown promise as a simple, cheap and effective means of reducing blood pressure among rural communities.

The perpetrators of the Chinese milk scandal are now facing death sentences, life imprisonment and multimillion dollar fines. Clearly, harsh penalties do not deter unscrupulous individuals from risking the well-being of others for personal profit, whether legally or illegally. The responsibility to protect the poor from these and other threats to their renal health lies with our greatest renoprotective agents-governments. Poverty might be inherited but it is not genetic. If we don't rise to this challenge, we are guilty not only of neglecting the most vulnerable members of society, but also of failing to address the most modifiable CKD risk factor of all.

doi:10.1038/nrneph.2009.66

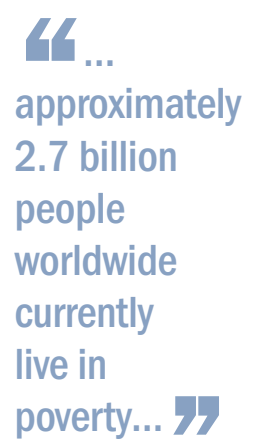

Chloë Harman is the Editor of Nature Reviews Nephrology.

Competing interests The author declared no competing interests. 